\title{
STATISTICAL PROPERTIES OF THE DISTANCE BETWEEN A TRAPPING CENTER AND A UNIFORM DENSITY OF DIFFUSING PARTICLES IN TWO DIMENSIONS
}

\author{
S. HAVLIN ${ }^{a, 1}$, H. LARRALDE ${ }^{b}$, R. KOPELMAN ${ }^{c}$ and G.H. WEISS ${ }^{a}$ \\ ${ }^{a}$ Physical Sciences Laboratory, National Institutes of Health, Bethesda, MD 20892, USA \\ ${ }^{b}$ Department of Physics, Boston University, Boston, MA 02215, USA \\ ${ }^{\mathrm{c}}$ Department of Chemistry, University of Michigan, Ann Arbor, MI 48109, USA
}

\author{
Received 6 September 1990
}

Several analyses of self-segregation properties of reaction-diffusion systems in low dimensions have been based on a simplified model in which an initially uniform concentration of point particles is depleted by reaction with an immobilized trap. A measure of selfsegregation in this system is the distance of the trap from the nearest untrapped particle. In one dimension the average of this distance has been shown to increase at a rate proportional to $t^{1 / 4}$. We show that this rate in a two-dimensional system is asymptotically proportional to $(\ln t)^{1 / 2}$, and that the concentration profile in the neighborhood of the trap is proportional to $(\ln r / \ln t)$.

There have been a number of recent studies of reaction-diffusion models in low dimensions because their kinetic properties can differ markedly from classical predictions. This phenomenon was first investigated by Ovchinnikov and Zeldovich [1] and later by Toussaint and Wilczek [2]. A number of additional references are given in reviews by Kopelman [3], and Kuzovkov and Kotomin [4]. In many formulations of these models it is possible to show that there may be self-segregation of the components, that is to say, there may be regions rich in one or the other of the reactants, with a corresponding depletion of the remaining reactants in other regions. A popular model which illustrates the general idea is based on the reaction $A+B \rightarrow B$. This may be thought of as the reaction of a molecule (A) with a perfectly efficient trap (B).

One example for such a reaction is the trapping of excitons. For instance, in a napthalene crystal with beta-methyl-napthalene impurities, a triplet exciton moves in a plane (ab) until trapped. An excitation pulse creates an initially random population of long-lived excitons which may decay primarily by

\footnotetext{
${ }^{1}$ Permanent address: Dept. of Physics, Bar-Ilan University, Ramat-Gan 52100, Israel.
} 
trapping and heterofusion at the trap [3]. Another example is a catalytic surface reaction in which the reaction, e.g., isomerization occurs at an active surface.

Following Smoluchowski $[5,6]$ one idealizes the reaction by assuming a single, immobile spherical B surrounded by an initially uniform distribution of A particles, each of which is allowed to diffuse, independent of the others, throughout an unbounded space. The occurrence of $\mathrm{A}-\mathrm{B}$ reactions creates a zone of depletion around the $B$ particle, which is one form of what is sometimes termed self-segregation. A quantitative characterization of this phenomenon can be framed in terms of one or more parameters. One of these is the distance of the $\mathrm{B}$ particle to the nearest unreacted $\mathrm{A}$. A second is the distance from the $B$ to the point at which the concentration reaches some specified fraction of its value in the bulk. If $\theta$ is the fraction we will refer to the distance as the $\theta$-distance.

Statistical properties for the first of these quantities have been studied in ref. [7] for the case of an immobile trap in one and three dimensions. In the one-dimensional example it has been shown that the mean distance from the $B$ to the ncarest unreacted $\mathrm{A}$ increases asymptotically with time as $\langle L\rangle \sim t^{1 / 4}$. It is trivial to show that the $\theta$-distance increases as $t^{1 / 2}$ for all values of $\theta$. In three dimensions both quantities are asymptotically constant in time, which means that self-segregation is minimal. In this note we consider the two-dimensional case in which the $B$ particle is taken to be a circle with a perfectly absorbing circumference. We will show that the distance from the circumference of the $B$ to the nearest unreacted $\mathrm{A}$ increases asymptotically like $(\ln t)^{1 / 2}$. More interestingly, we will show that in two dimensions the $\theta$-distance has no universal scaling but rather depends on the value of $\theta$.

We will assume cylindrical symmetry in the system in which the concentration of $\mathrm{A}$ 's at a distance $r$ from the origin will be denoted by $c(r, t)$. This function will be assumed to satisfy an ideal diffusion equation with diffusion constant $D$, which is to be solved subject to the initial condition $c(r, 0)=c_{0}-$ constant, and the boundary condition $c(a, t)=0$, where $a$ is the radius of the circle. The solution to this problem is well known and is found, for example, in ref. [8]. It can be expressed in the form $c(r, t)=c_{n} g(r, t)$, where

$$
g(r, t)-\frac{2}{\pi} \int_{0}^{c} \operatorname{cxp}\left(-D t u^{2}\right) \frac{J_{0}(a u) Y_{0}(r u)-J_{0}(r u) Y_{0}(a u)}{J_{0}^{2}(a u)+Y_{0}^{2}(a u)} \frac{\mathrm{d} u}{u} .
$$

We will be interested in the form of this function in the limit $t \rightarrow \infty$. The principal contribution to the integral then comes from small $u$. In this limit we may replace the numerator of the integral in eq. (1) by

$$
J_{0}(a u) Y_{0}(r u)-J_{0}(r u) Y_{0}(a u) \sim \frac{2}{\pi} \ln \left(\frac{r}{a}\right),
$$


which, together with eq. (1), implies that

$$
g(r, t) \sim \frac{4}{\pi^{2}} \ln \left(\frac{r}{a}\right)\left[\frac{1}{\ln \left(\frac{4 D t}{a^{2}}\right)-2 \gamma}-\frac{\gamma}{\left[\ln \left(\frac{4 D t}{a^{2}}\right)-2 \gamma\right]^{2}} \cdots\right]
$$

provided that $r^{2} \ll D t$, where $\gamma$ is Euler's constant, equal to $0.577+$. Thus we see that in the long-time limit $g(r, t)$ factors into a product of two functions, one depending only on $r$ and the second only on $t$, i.e., $g(r, t) \sim \ln (r / a) F(t)$ where $F(t)$ represents the bracketed terms in eq. (3). From this we can infer that the flux into the circle is

$$
-J(t)=\left.\frac{D}{a} \frac{\partial c}{\partial r}\right|_{r=a}=\frac{D}{a^{2}} F(t),
$$

which means that at very long times it is proportional to $(\ln t)^{-1}$.

The relation in eq. (2) allows us to calculate the $\theta$-distance from the cquation $g\left(r_{\theta}, t\right)=\theta$ with a solution of the form

$$
\ln \left(\frac{r_{\theta}}{a}\right)=\frac{\theta}{2 F(t)} .
$$

Retaining only the lowest order term in the expansion of $F(t)$ we find that

$$
r_{\theta} \sim a\left(\frac{4 D t}{a^{2}}\right)^{\theta / 2},
$$

which means that the exponent in the asymptotic scaling law depends on $\theta$.

Finally, the asymptotic form of the probability density for the distance from the origin to the nearest untrapped A can be found following the analysis in ref. [7]. Let $Q(L, t)$ be the probability that the nearest neighbor distance is less than or equal to $L$. The probability density of the nearest neighbor distance, $f(L, t)$, is then given by $-\partial Q / \partial L$. But $Q(L, t)$ is given by

$$
Q(L, t)=\exp \left(-2 \pi c_{0} \int_{a}^{L} g(r, t) r \mathrm{~d} r\right) .
$$

The asymptotic form of this function is found by inserting the approximation in eq. (2) into this relation. This sequence of operations leads to the result

$$
f(L, t) \sim 2 \pi c_{0} L\left[2 \ln \left(\frac{L}{a}\right)+1\right] F(t) \exp \left\{-2 \pi c_{0} F(t)\left[L^{2} \ln \left(\frac{L}{a}\right)-\frac{a^{2}}{4}\right]\right\} .
$$




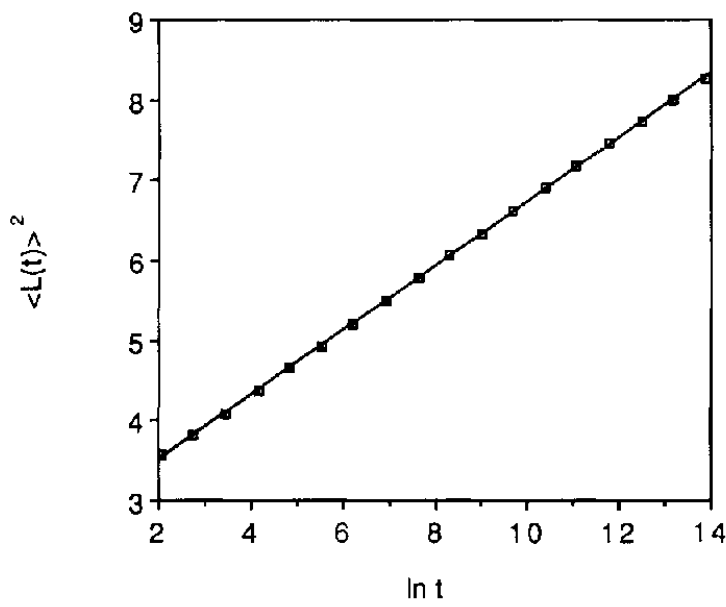

Fig. 1. Plot of $\langle L(t)\rangle^{2}$ versus $\log t$ obtained from the exact cnumeration method [9|. The linear curve supports the theoretical prediction, eq. (9).

From this it follows that the asymptotic time dependence of $\langle L\rangle$, the mean distance to the nearest untrapped $\mathrm{A}$, has the form

$$
\langle L(t)\rangle \times(\ln t)^{1 / 2}
$$

and the variance, $\sigma^{2}(L) \equiv\left\langle L^{2}\right\rangle-\langle L\rangle^{2}$, is asymptotically proportional to $\ln t$. Fig. 1, derived from the method of exact enumeration [9], lends numerical support to this prediction.

A much harder problem relates to properties of the nearest neighbor distances when both the trap and the particles are allowed to diffuse. In this case results for this type of system can only be derived numerically [10], although an approximation scheme for the survival probability in such a system is given in ref. [11].

\section{Note added in proof}

Since obtaining our results we have found that S. Redner and D. benAvraham have found similar results using a somewhat different approach in an as yet unpublished manuscript.

\section{Acknowledgements}

H. Larralde acknowledges support by CONACYT (Mexico) by Grant 57312 . 
The work of S. Havlin and G.H. Weiss was supported in part by the US-Israel Binational Science Foundation.

\section{References}

[1] A.A. Ovchinnikov and Y.B. Zeldovich, Chem. Phys. 28 (1978) 215.

[2] D. Toussaint and F. Wilczek, J. Chem. Phys. 78 (1983) 2642.

[3] R. Kopelman, Science 241 (1988) 1620.

[4] V. Kuzovkov and E. Kotomin, Rep. Prog. Phys. 51 (1988) 1479.

[5] M. von Smoluchowski, Z. Phys. Chem. 92 (1917) 129.

[6] S.A. Rice, Diffusion-Limited Reactions (Elsevier, Amsterdam, 1985).

[7] G.H. Weiss, R. Kopelman and S. Havlin, Phys. Rev. A 39 (1989) 466.

[8] H.S. Carslaw and J.C. Jaeger, Conduction of Heat in Solids, 2nd ed. (Clarendon, Oxford, 1959).

[9] S. Havlin and D. ben-Avraham, Adv. Phys. 36 (1987) 695.

[10] R. Schoonover, D. ben-Avraham, S. Havlin, R. Kopelman and G.H. Weiss, Physica A (to appear).

[11] A. Szabo, R. Zwanzig and N. Agmon, Phys. Rev. Lett. 61 (1988) 2496. 